\title{
La selección de lugares por apicultores de Costa Rica es influenciada por el uso de terreno, sus recursos florales y la calidad de la carretera ${ }^{1}$
}

\author{
Héctor S. Tavárez ${ }^{2, *}$, Sara M. Galbraith ${ }^{3}$ \\ y Nilsa A. Bosque-Pérez ${ }^{4}$
}

J. Agric. Univ. P.R. 102 (1-2):21-37 (2018)

\begin{abstract}
RESUMEN
Los humanos obtienen múltiples servicios de los ecosistemas, valorados en billones de dólares anualmente. A pesar de la importancia de estos servicios alrededor del mundo, aún seguimos enfrentando cambios en la cobertura y uso de terreno que pueden impactar negativamente la provisión de los servicios ecosistémicos. El objetivo de este estudio fue examinar cómo las características de un lugar afectan la selección de terrenos para practicar la apicultura. Desarrollamos un cuestionario con preguntas cerradas y utilizamos un Modelo Logit Ordenado para evaluar cómo las características de la región afectan la toma de decisiones de los apicultores en la Península de Nicoya, Costa Rica. Los resultados demuestran que aumentos en la cantidad de bosque, pasto o café, aumentan la probabilidad de seleccionar una opción como mejor alternativa para colocar las colmenas de abejas, siendo el bosque el uso de terreno preferido por la diversidad de recursos florales. Los resultados también demuestran que los lugares con acceso a mejores carreteras tienen mayor probabilidad de ser seleccionados como mejor alternativa para colocar las colmenas.
\end{abstract}

Palabras clave: cambio de uso de terreno, servicios ecosistémicos, Modelo Logit Ordenado, apicultura, Costa Rica

\section{ABSTRACT \\ Beekeepers site selection in Costa Rica is influenced by land use, floral resources and road quality}

Humans obtain multiple services from ecosystems, valued at trillions of dollars annually. Despite the importance of these services worldwide,

${ }^{1}$ Manuscrito sometido a la Junta Editorial el 28 de febrero de 2018.

${ }^{2}$ Catedrático Auxiliar, Departamento de Economía Agrícola y Sociología Rural, Colegio de Ciencias Agrícolas, Universidad de Puerto Rico - Estación Experimental Agrícola, Jardín Botánico Sur, 1193 Calle Guayacán, San Juan, Puerto Rico 00926-1118. *Autor para correspondencia: hector.tavarez2@upr.edu

${ }^{3}$ Asociada Postdoctoral de Investigación, Departamento de Ecosistemas Forestales y Sociedad, Universidad del Estado de Oregón, Oregón, Estados Unidos.

${ }^{4}$ Catedrática, Departamento de Entomología, Fitopatología y Nematología, Universidad de Idaho, Moscow, Idaho, Estados Unidos. Programa de Ciencias Ambientales, Universidad de Idaho, Moscow, Idaho, Estados Unidos. 
land-use and land cover changes occurring have the potential to negatively impact ecosystems services. The objective of this study was to examine how site characteristics affect site selection for beekeeping. We developed a questionnaire with closed-ended questions and used an Ordered Logit Model to evaluate the effects that changes in specific factors can have on beekeepers' decision-making process in the Nicoya Peninsula of Costa Rica. Results demonstrate that increasing the quantity of forest, pasture and coffee lands increase the probability of selecting a site scenario as the best option for hive placement, with the forest as the preferred land use due to the diversity of floral resources. Results also show that sites with better roads have higher probabilities of being selected as the best option for hive placement.

Key words: land use change, ecosystem services, Ordered Logit Model, apiculture, Costa Rica

\section{INTRODUCCIÓN}

Los servicios que proveen los ecosistemas, o servicios ecosistémicos, son los beneficios que las personas obtienen de la naturaleza (de Groot et al., 2002; MEA, 2005). Por ejemplo, los bosques proveen beneficios directos e indirectos, tales como el secuestro de carbono, belleza escénica y purificación del agua. Además, los bosques proveen hábitat para insectos polinizadores, afectando la cantidad y calidad de ciertos cultivos en zonas aledañas al bosque (Ricketts, 2004). Por esta razón, los cambios en la cobertura y uso de terreno alteran la disponibilidad de los servicios ecosistémicos y, consecuentemente, el sistema socialecológico de una región (Ostrom, 2009). La apicultura es un sector de la economía que se puede afectar por cambios en la cobertura y uso de terreno, debido a cambios en los recursos florales disponibles para las abejas. Con la población de polinizadores disminuyendo a nivel mundial y el aumento en los colapsos de la población de abejas melíferas, Apis mellifera (Steffan-Dewenter et al., 2005; Chagnon, 2008; Potts et al., 2010) es de suma importancia entender cómo las actividades antropogénicas afectan a los polinizadores. En este estudio, analizamos cómo los cambios de uso de terreno y otras características de la región pueden afectar la selección de lugares para practicar la apicultura.

Los cambios en la cobertura y uso de terreno a menudo son incentivados por políticas o estrategias de conservación a nivel nacional. Un ejemplo de esto es Costa Rica, un país que ha sido pionero en el programa de Pagos por Servicios Ambientales (PSA) (Pagiola, 2008; Pattanayak et al., 2010). Este programa otorga compensaciones a los dueños de terrenos que ayudan a proteger los servicios ecosistémicos por medio del manejo de sus tierras (Tavárez, 2016). Algunas de las opciones para el manejo de tierras reconocidas por el PSA en Costa Rica son la conservación de bosques, reforestación con especies nativas y plantación con especies no nativas. El cambio en la cobertura y uso 
de terreno en Costa Rica ha estado sujeto a modificaciones durante las últimas décadas debido en parte al PSA (Calvo-Alvarado et al., 2009).

Las abejas melíferas están declinando mundialmente por una variedad de razones, tales como: (1) el aumento en el uso de plaguicidas, (2) la pérdida de hábitat, (3) el aumento en enfermedades y plagas, y (4) el cambio climático (Chagnon, 2008; Potts et al. 2010; Stokstad, 2012). La disminución de polinizadores tiene muchas implicaciones, por ejemplo, los servicios de polinización son insumos para muchos cultivos agrícolas. Consecuentemente, la reducción de abejas melíferas puede impactar las ganancias de muchas industrias agrícolas (Kasina et al., 2009; Potts et al., 2010), así como la seguridad alimentaria y la función de los ecosistemas (Vanbergen, 2013). Otro beneficio de los servicios de polinización es que ayudan a mantener las plantas silvestres y los recursos florales y, consecuentemente, ayudan a mantener la biodiversidad (Potts et al., 2010). Las abejas son críticas para el mantenimiento de los ecosistemas (Hilmi et al., 2011).

A pesar de que Costa Rica no es uno de los mayores productores de miel en América Latina, la apicultura juega un papel importante en la economía local. Entre el 2008 y el 2011, la producción de miel en Costa Rica aumentó en un 66\% (Brenes-Quirós, 2012). En las comunidades que forman parte de las zonas rurales, donde los ingresos son limitados, la apicultura puede contribuir a la seguridad de los medios de subsistencia (Bradbear, 2009; Hilmi et al., 2011). Además, la apicultura provee ingresos que no requieren deforestación ni destrucción de recursos naturales. Las abejas melíferas no solamente producen miel, también contribuyen a la generación de ingresos adicionales por medio de renta para polinización y otros productos derivados de la apicultura, como la cera y jabones, que pueden contribuir al sustento de vida de las familias. Sin embargo, los cambios en la cobertura y uso de terreno han impactado en múltiples maneras la disponibilidad de recursos florales en algunas regiones de Costa Rica, afectando la apicultura local (Galbraith et al., 2017). Debido a estos cambios, urge realizar estudios que contribuyan a entender los factores que afectan la subsistencia de los apicultores, especialmente en zonas que han sido marginadas históricamente.

Entender cómo los cambios en la cobertura y uso de terreno afectan a las abejas y a la apicultura es de suma importancia para el desarrollo de la economía local y la conservación de la biodiversidad. Por ejemplo, es posible que los apicultores perciban que las fincas de café (Coffea arabica) que integran árboles con especies nativas, proveen mayores recursos florales que aquellas fincas con plantaciones de teca (Tectona grandis), la cual, a pesar de ser una especie no-nativa de la región, califica para el programa de PSA en Costa Rica. Existen otros factores que pueden afectar la selección de terrenos, tales como la calidad de 
la infraestructura y características socio-demográficas del apicultor, tales como el ingreso, educación, y edad. Estos factores ambientales y socio-demográficos pudieran afectar la selección del terreno donde los apicultores deciden colocar sus colmenas. Entender las preferencias y el comportamiento de los apicultores hacia la selección de terrenos bajo diferentes coberturas y uso de terreno, puede aportar a un mejor entendimiento sobre los impactos del cambio de uso de terreno. Este conocimiento puede proveer información valiosa para el desarrollo de políticas con el objetivo de contribuir a la economía local y al manejo de los ecosistemas. Sin embargo, actualmente se sabe muy poco sobre las características específicas que debe tener un lugar para que sea atractivo para la ubicación de colmenas de abejas por apicultores.

El objetivo general de este estudio es contribuir al entendimiento del impacto de las estrategias y políticas de conservación sobre las abejas y apicultores. El objetivo específico de este estudio fue examinar cómo las características de un lugar afectan la selección de terrenos para practicar la apicultura. Para cumplir con los objetivos de este estudio, desarrollamos un cuestionario con preguntas cerradas y utilizamos un Modelo Logit Ordenado (MLO).

\section{MATERIALES Y MÉTODOS}

Área de estudio

Este estudio se realizó en los condados de Puntarenas, Nandayure, Hojancha y Nicoya, los cuales forman parte de la provincia de Guanacaste en Costa Rica. Esta región ha experimentado aumentos en la producción de miel durante los últimos años. Para el 2012, se estimaba que el 57\% de las colmenas en Costa Rica estaban ubicadas en la provincia de Guanacaste (Brenes-Quirós, 2012). Los condados que forman parte de este estudio se encuentran ubicados en el suroeste de Guanacaste en la Península de Nicoya. Actualmente existen dos asociaciones de apicultores en esta región, la Asociación de Apicultores de Jicaral y la Asociación de Apicultores de Chorrotega. Solo el 24\% de los apicultores no son miembros de estas asociaciones (Galbraith et al., 2017).

La cobertura y uso de terreno en esta región ha cambiado drásticamente durante las últimas décadas (Vallejo et al., 2006), lo cual ha alterado la provisión de servicios ecosistémicos. La región experimentó una deforestación masiva durante las décadas del cincuenta y sesenta para expandir el mercado del sector ganadero. Posteriormente, se abandonó el sector ganadero en la década del setenta por colapsos en el precio de la carne y se expandió el sector cafetalero. Años más tarde surge un interés por desarrollar plantaciones de teca para estimular 
la economía local. Acción local por parte de actores claves e iniciativas desarrolladas por el Centro Agrícola Cantonal de Hojancha $(\mathrm{CACH})$ contribuyeron al aumento de plantaciones de teca. A partir de los noventa Costa Rica realiza considerables esfuerzos para aumentar la cobertura de bosque en la región y el país. La cobertura de bosque en la Península de Nicoya ha aumentado durante las últimas décadas, pero el porcentaje de tierras agrícolas ha disminuido (Serrano, 2005; Vallejo et al., 2006). Cada uno de estos usos de terreno proveen múltiples servicios ecosistémicos a diferentes niveles (Daily et al., 2009), lo cual puede alterar el sistema social-ecológico de la región (Ostrom, 2009).

\section{Recopilación de datos}

Desarrollamos un cuestionario para la recopilación de datos ${ }^{5}$, los cuales se obtuvieron de marzo a noviembre del 2014. Los cuestionarios se distribuyeron en persona a cincuenta apicultores establecidos en los condados de Puntarenas, Nandayure, Hojancha y Nicoya. Estos apicultores fueron identificados por la Asociación de Apicultores de Jicaral $(n=21)$ y la Asociación de Apicultores de Chorrotega $(n=17)$. El resto de los apicultores no estaban afiliados a una asociación y fueron identificados por referidos en cadena $(n=12)$. A pesar de no conocer el número total de apicultores en la región, nuestra estrategia de muestreo nos brinda la seguridad que se entrevistó la gran mayoría de los apicultores en la región debido a que las asociaciones de apicultores de Jicaral y Chorrotega son las únicas dos asociaciones en la región. Además, nosotros continuamos las entrevistas hasta que no conseguimos más apicultores por referidos en cadena (Galbraith et al., 2017).

El cuestionario estuvo compuesto por dos secciones ${ }^{6}$. El orden de estas secciones fue alternado entre los apicultores. Una sección del cuestionario se utilizó para obtener información a ser utilizada en el MLO. La otra sección del cuestionario se utilizó para recopilar información sobre las características socio-demográficas de los participantes, tales como el ingreso del hogar, nivel de educación, número de dependientes y número de apiarios. La información recopilada en esta sección del cuestionario fue utilizada para entender mejor las respuestas en el MLO, preferencias de los apicultores sobre las características alrededor de un apiario y el impacto de los cambios de uso de terreno sobre el sustento de vida de los apicultores. Las preguntas utilizadas para entender mejor las preferencias de los apicultores sobre las características alrededor de un

${ }^{5}$ Los participantes también fueron entrevistados utilizando preguntas semi-estructuradas y completaron un ejercicio de trazado de mapa. Sin embargo, la información recopilada de las entrevistas semi-estructuradas y el trazado de mapa fue utilizada para cumplir con objetivos que fueron publicados en otro estudio.

${ }^{6} \mathrm{El}$ cuestionario está disponible de ser solicitado. 
apiario y uso de terreno fueron cerradas tipo Escala-Likert (1=Nada importante, $5=$ Extremadamente importante). Los participantes tuvieron la oportunidad de hacer comentarios al final del cuestionario.

\section{Diseño experimental - $M L O$}

Para recopilar información sobre las preferencias de los apicultores sobre los usos de terreno y examinar cómo el uso de los terrenos afecta la selección de lugares para colocar las colmenas, cada apicultor recibió seis escenarios compuestos por cuatro opciones. Cada opción, a su vez, estaba compuesta por tres factores principales (Figura 1). Los factores en cada opción fueron los siguientes: (1) uso de terreno en los alrededores, (2) calidad de la carretera y, (3) distancia del hogar. Luego de explicarle a cada apicultor la naturaleza del ejercicio, se les solicitó que ordenaran las opciones desde la más preferida hasta la menos preferida.

Uno de los pasos más importantes en todo estudio es definir cada variable de interés, de esta forma todos los participantes pueden entender el significado de cada factor. Las expectativas del estudio era que todos los participantes interpretaran cada factor de forma homogénea. De lo contrario, los participantes podrían proveer diferentes resultados dependiendo de su propia interpretación de las variables. Los factores que pueden afectar la decisión de los apicultores para la ubicación de colmenas fueron seleccionados basados en una revisión de la literatura, reuniones con expertos y entrevistas preliminares a apicultores con varios años de experiencia. A continuación, se presentan los factores seleccionados.

\section{A. Uso de terreno en los alrededores (indicador de recursos florales)}

Este factor se refiere al tipo de uso de terreno alrededor del lugar identificado para colocar las colmenas. Utilizamos un círculo con radio de dos kilómetros porque se ha reportado que esta puede ser la distancia promedio que las abejas viajan para tener acceso a los recursos florales y al agua (Abou-Shaara, 2014), aunque pueden viajar distancias más largas dependiendo del tamaño del insecto, estación y tipo de vegetación (Greenleaf et al., 2007; Couvillon et al., 2015). Toda la información sobre los diferentes usos de terreno fue presentada en forma escrita y con fotos para el mejor entendimiento de los apicultores. Los usos de terreno son definidos a continuación? ${ }^{7}$ :

\footnotetext{
${ }^{7}$ Las plantaciones con teca no se incluyeron debido a que en este estudio se busca entender cuáles son los usos de terrenos que afectan positivamente la selección de lugares para la apicultura. En entrevistas informales con actores claves se nos informó que las plantaciones con teca no son de interés en lo absoluto para los apicultores. El impacto negativo de la teca sobre los apicultores fue evaluado con más detalle en Galbraith et al. (2017).
} 
Por favor, compare las ventajas y desventajas en cada lugar (opción) y ordénelos desde el lugar más preferido hasta el menos preferido. Los círculos abajo representan la distribución de la cobertura de terrenos alrededor de donde se encuentran las colmenas.


época de lluvia o seca

-Distancia desde el hogar- 5 kilómetros
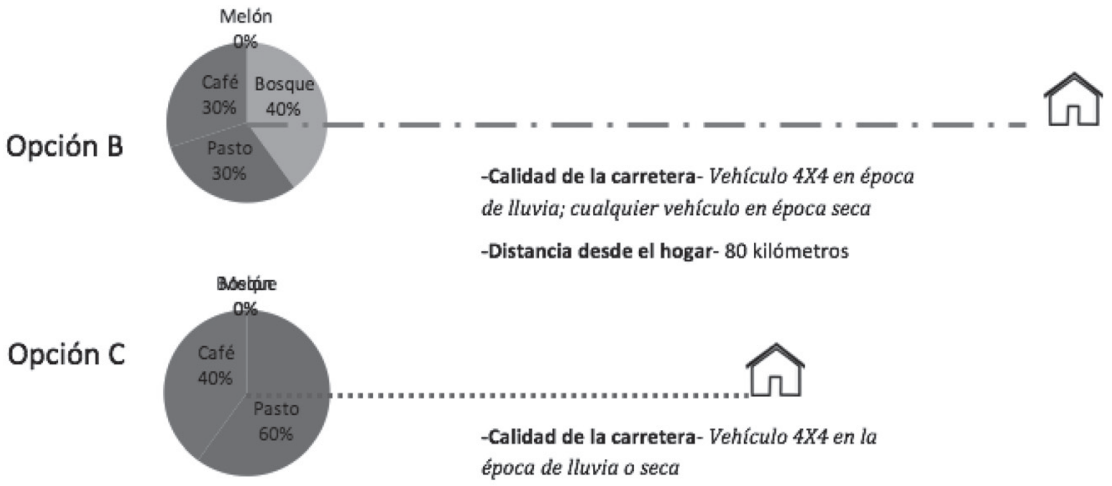

-Distancia desde el hogar- 25 kilómetros

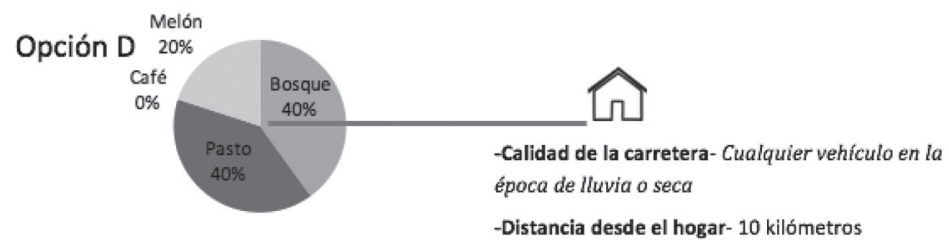

Si estas fueran las únicas opciones para la selección de lugar, ¿como usted ordenaría las alternativas desde la más preferida hasta la menos preferida?

Más preferido: Opción

$2^{\text {do }}$ más preferido: Opción

$3^{\text {ro }}$ más preferido: Opción

Menos preferido: Opción

FiguRA 1. Ejemplo de escenario donde los participantes analizaron y ordenaron cada alternativa, según sus preferencias. Esta figura es un resumen de los escenarios que recibieron los participantes. Los escenarios que recibieron los participantes estaban compuestos por cuatro opciones.

a. Bosque Nativo - área de terreno dominada por árboles nativos y con muy poco o nada de manejo humano. Por lo tanto, bajo esta definición las plantaciones de teca no son consideradas como 
bosque. Los porcentajes de bosque nativo alrededor del apiario fueron los siguientes: $60 \%, 40 \%, 30 \%, 20 \%, 10 \%, 0 \%{ }^{8}$.

b. Pasto - área de terreno dominada por hierba, usualmente utilizada para ganadería. Los porcentajes de pasto alrededor del apiario fueron los siguientes: $60 \%, 40 \%, 30 \%, 20 \%, 10 \%, 0 \%$.

c. Café - área utilizada para la producción de café. Se refiere a las fincas promedio de café en la región, las cuales tienen algunos árboles proveyendo sombra. Los porcentajes de café alrededor del apiario fueron los siguientes: 40\%, 30\%, 20\%, 10\%, $0 \%$.

d. Melón - área utilizada para la producción de melones. Los porcentajes de melón alrededor del apiario fueron los siguientes: $40 \%, 30 \%, 20 \%, 10 \%, 0 \%$.

\section{B. Calidad de la carretera}

Este factor se refiere a la calidad de la carretera utilizada para acceder al lugar identificado para colocar las colmenas. La infraestructura vial puede ser crucial para los apicultores porque les permite tener acceso al mercado y alcanzar mejores precios (Bradbear, 2009). Sin embargo, algunos apicultores de la región han argumentado que mejorar las carreteras puede ser un indicador de inestabilidad debido a que facilita la expansión agrícola y urbana. Para examinar las preferencias por la calidad de la carretera utilizamos como indicador el tipo de vehículo que puede acceder al lugar durante las diferentes épocas del año. Los tipos de carretera (niveles de calidad) son definidos a continuación:

a. Buena - Cualquier vehículo tiene acceso al lugar durante la época seca o lluviosa.

b. Regular - Solo vehículos 4x4 tienen acceso al lugar durante la época lluviosa, pero cualquier vehículo tiene acceso al lugar durante la época seca.

c. Mala - Solo vehículos $4 \mathrm{x} 4$ tienen acceso al lugar durante la época seca o lluviosa.

Para facilitar la interpretación de la calidad de carretera también utilizamos diagramas donde la calidad de la carretera fue explicada con dibujos (Figura 1). El objetivo de este método fue facilitar el entendimiento de la calidad de carretera entre los participantes.

${ }^{8}$ De acuerdo al Atlas de Costa Rica, alrededor de $60 \%$ de la cobertura de terreno en la Península de Nicoya es bosque. 
Para la calidad de carretera considerada Buena, utilizamos líneas continuas. Para la calidad de carretera considerada Regular, utilizamos una combinación de puntos y líneas entrecortadas. Finalmente, para calidad de carretera considerada Mala, utilizamos puntos continuos.

\section{Distancia del hogar}

Este factor se refiere a la distancia desde la casa donde reside el apicultor al lugar identificado para colocar las colmenas. Las distancias utilizadas fueron las siguientes: $5 \mathrm{~km}, 10 \mathrm{~km}, 25 \mathrm{~km}, 50 \mathrm{~km}$ y $80 \mathrm{~km}$.

\section{Datos y modelo de estimación}

Para analizar los datos colectados utilizamos análisis descriptivo y el MLO, el cual ha sido utilizado cuando los valores de la variable dependiente indican un orden (Long and Freese, 2001; MadrigalBallestero et al., 2013; Kemperman et al., 2003). Contrario a los modelos logit y probit donde la variable dependiente es binaria, la variable dependiente en el MLO tiene más de dos categorías. Además, estas categorías tienen un orden secuencial, donde el valor de una categoría es "mayor" al valor de la anterior. El MLO nos permite evaluar la probabilidad de pertenecer a una categoría en particular, contrario al modelo logit o probit que estiman la probabilidad de que suceda un evento o acción. Los coeficientes en modelos logit son estimados utilizando el procedimiento de máxima verosimilitud (Kemperman et al., 2003).

Nuestra meta fue examinar cómo las características de un lugar afectan la selección de terrenos para practicar la apicultura. Utilizamos el MLO para examinar la significancia y signos de los coeficientes de cada uno de los factores utilizados en este estudio. Posteriormente, estimamos los efectos marginales de cada factor en el MLO para entender mejor la probabilidad de seleccionar un lugar para la apicultura cuando cambia la cantidad de terreno en bosque, pasto y café en los alrededores, y cuando cambia la calidad de la carretera hacia el lugar. Los factores asociados al uso de terreno y la distancia hasta el hogar fueron especificados en el modelo como variables continuas, mientras el factor asociado a la calidad de carretera fue especificado en forma binaria. El factor Melón no fue especificado en el MLO final debido a que existe una alta multicolinealidad con los otros usos de terreno, pues en términos porcentuales suman uno. El porcentaje de hectáreas a un radio de $2 \mathrm{~km}$ alrededor del lugar de cada uso de terreno fue convertido a kilómetros cuadrados para analizar los datos del MLO. Esto permitió que la interpretación de los resultados sea más tangible. 


\section{RESULTADOS Y DISCUSIÓN}

Análisis descriptivo de los apicultores

Los participantes que formaron parte de este estudio tenían en promedio 50.7 años de edad y llevaban 18.1 años practicando la apicultura (Cuadro 1). De estos, el 90\% son hombres. El 58\% de los apicultores no habían completado la escuela secundaria y solo el $16 \%$ tenía un grado de bachillerato universitario o más alto. El número promedio de dependientes por apicultor era de 2.4. El 54.1\% de los ingresos del hogar eran obtenidos por ingresos relacionados a la apicultura. Por último, los apicultores tenían en promedio 5.8 apiarios y 27.6 colmenas por apiario.

\section{Percepción sobre las características del lugar alrededor del apiario ${ }^{9}$}

Identificamos varios factores relacionados a las preferencias por el uso de terreno e infraestructura vial que nos ayudaron a entender mejor las contestaciones en el MLO. En preguntas cerradas, los apicultores mencionaron que las características más importantes que debe tener un lugar para colocar las colmenas son la distancia a fuentes de agua y el uso de terreno en los alrededores, aunque también mencionaron que la diversidad de árboles en la región es importante (Galbraith et al., 2017). Algunos apicultores también

CuADRo 1.-Características socio-demográficas de los participantes.

\begin{tabular}{|c|c|c|c|}
\hline Características & Descripción & Promedio & $(\mathrm{DE})$ \\
\hline Edad & Edad del participante & 50.7 & 11.56 \\
\hline Años de apicultor & $\begin{array}{l}\text { Años del participante trabajando en } \\
\text { la apicultura }\end{array}$ & 18.1 & 12.42 \\
\hline Sexo & $1=$ hombre, $0=$ mujer & 0.9 & 0.30 \\
\hline Educación ${ }^{1}$ & $\begin{array}{l}\text { Educación del participante }(1=\text { primaria, } \\
4=\text { grado de bachillerato o más alto })\end{array}$ & 1.8 & 1.13 \\
\hline Dependientes $^{2}$ & Número de dependientes en el hogar & 2.4 & 1.47 \\
\hline Apicultura/Ingreso & $\begin{array}{l}\text { Porcentaje del ingreso que viene de } \\
\text { la apicultura }\end{array}$ & 54.1 & 38.80 \\
\hline Apiarios & Número de apiarios por participante & 5.8 & 4.88 \\
\hline Colmenas & Número de colmenas por apiario & 27.6 & 2.74 \\
\hline
\end{tabular}

DE - Desviación Estándar

${ }^{1}$ La descripción de las categorías de Educación es la siguiente: 1 = Primaria, 2 = Secundaria, 3 = Créditos universitarios, 4 = Grado de bachillerato universitario o más alto.

${ }^{2}$ Personas que dependen del $50 \%$ o más del ingreso del apicultor.

${ }^{9}$ Los detalles sobre las preferencias de los apicultores por el uso de terreno alrededor del lugar identificado para colocar las colmenas y por las especies de árboles preferidas para la apicultura fueron evaluados cualitativamente y discutidos en otro estudio (Galbraith et al., 2017). Por esta razón, no se discute en profundidad en este estudio. 
mencionaron que la distancia a áreas residenciales y área donde se utiliza plaguicida o herbicida son otras características a considerar cuando se va a seleccionar un lugar para colocar las colmenas. Además, los apicultores mencionaron en preguntas de seguimiento que es importante considerar los usos del terreno en los alrededores a una distancia en promedio de $2.1 \mathrm{~km}(\mathrm{DE}=1.2)$. Este es un hallazgo importante porque en los escenarios utilizados para el análisis del MLO se asumió, basado en información obtenida en revisión de literatura (Bradbear, 2009; Abou-Shaara, 2014), que las abejas pueden viajar en promedio alrededor de $2 \mathrm{~km}$ para obtener sus recursos florales. Tener alineado la percepción de los apicultores con la presunción acogida sobre el rango de forrajeo en los escenarios para el MLO es crítico debido a que contribuye a la credibilidad del método, lo cual es importante para obtener mejores resultados (Arrow et al., 1993; Bateman et al., 2002).

Uno de los factores que recibió menos puntuación en preguntas tipo Escala-Likert fue la distancia desde el hogar al lugar identificado para colocar las colmenas. Este resultado puede sorprender debido a que, a mayor distancia, mayores serán los costos directos asociados a la apicultura. Sin embargo, los beneficios y costos marginales pueden explicar por qué se calificó la distancia desde el hogar al lugar identificado para colocar las colmenas como uno de los factores menos importantes. Si los costos de transportación son pequeños, relativo a los ingresos, los apicultores pudieran enfocarse en otros factores que afectan directa o indirectamente la producción de miel, debido a que estos factores pudieran disminuir las ganancias en mayor magnitud. En este caso, la distancia puede considerarse menos importante que otros factores que afectan a las abejas y, consecuentemente, la producción de miel. Otro factor que recibió menos puntuación fue el pago mensual (i.e., renta) al dueño del terreno. La gran mayoría de los apicultores no son dueños de las fincas donde colocan sus colmenas. Sin embargo, los apicultores actualmente no pagan con dinero en efectivo por utilizar terrenos aledaños a bosque para practicar la apicultura. En algunos casos, ellos compensan al propietario con 10 a 14 galones de miel cruda. Por lo tanto, el pago mensual al propietario no es un factor importante porque existen alternativas para compensar al dueño del terreno. Por último, la calidad de la carretera también fue uno de los factores que recibió menos puntuación. Según reportaron los apicultores en preguntas de seguimiento, el 50\% de los apicultores cuenta con vehículos 4x4 para realizar sus actividades relacionadas a la apicultura. Además, algunos apicultores mencionaron que pueden conseguir estos vehículos prestados de ser necesario. 


\section{Resultados del Modelo Logit Ordenado}

Todas las variables explicativas del MLO son estadísticamente significativas (Cuadro 2) ${ }^{10}$. El modelo ajusta los datos con un Pseudo de 0.12. El signo de la variable DISTANCIA es negativo, sugiriendo que aumentos en la distancia del hogar al lugar reduce la probabilidad de seleccionar una opción como mejor alternativa para colocar las colmenas. El signo de las variables BOSQUE, PASTO y CAFE es positivo, indicando que aumentos en la cantidad de área de estos usos de terreno, aumenta la probabilidad de seleccionar una opción como mejor alternativa para colocar las colmenas. Por último, el signo de las variables relacionadas a la calidad de la carretera, CARRETERA_1 y CARRETERA_2, son positivos, sugiriendo que áreas con mejores carreteras tienen mayor probabilidad de ser seleccionadas como mejor alternativa para colocar las colmenas.

Las características socio-demográficas de los participantes también fueron incluidas en el MLO como variables explicativas. Sin embargo, estas no fueron significativas y solo aumentaron el Pseudo por 0.01. Por lo tanto, la contribución de estas variables al modelo es menor. Estos resultados indican que las características socio-demográficas como la edad, ingreso y educación de los apicultores en la región no afectan las preferencias por los lugares identificados para colocar las colmenas. Otras variables de percepción sobre las preferencias de uso de

CuAdro 2.-Resultados del Modelo Logit Ordenado.

\begin{tabular}{lccccc}
\hline Variables & Coeficiente & $\mathrm{EE}$ & Valor Z & $\operatorname{Pr}(>|\mathrm{z}|)$ & $\mathrm{IC}^{1}(95 \%)$ \\
\hline BOSQUE & 0.478 & 0.044 & 10.89 & $0.000^{* 2}$ & $0.392,0.564$ \\
PASTO & 0.111 & 0.041 & 2.74 & $0.006^{*}$ & $0.032,0.192$ \\
CAFÉ & 0.114 & 0.031 & 3.66 & $0.000^{*}$ & $0.053,0.175$ \\
CARRETERA_1 & 0.247 & 0.139 & 1.77 & $0.076^{* *}$ & $-0.0259,0.520$ \\
CARRETERA_2 & 0.547 & 0.140 & 3.92 & $0.000^{*}$ & $0.273,0.820$ \\
DISTANCIA & -0.010 & 0.001 & -9.50 & $0.000^{*}$ & $-0.0121,-0.008$ \\
N & 1,072 & & & & \\
Pseudo R & 0.12 & & & & \\
Prob $>\chi^{2}$ & 0.000 & & & & \\
\hline
\end{tabular}

${ }^{1} \mathrm{IC}=$ Intervalo de confianza

$2 *$ Significativo al $0.01 ; * *$ Significativo al 0.1

${ }^{3}$ CARRETERA_1 = lugares con carreteras que se consideran Regular

${ }^{4}$ CARRETERA_2 = lugares con carreteras que se consideran Buenas

${ }^{10}$ Utilizamos también otras especificaciones de modelos para estudiar la significancia y dirección de las variables. Por ejemplo, utilizamos un modelo logit creando otra variable dependiente ( 1 para aquellos que contestaron 4 y 0 de lo contrario) y una regresión múltiple. A pesar de utilizar modelos con especificaciones diferentes, la significancia y dirección de las variables se mantuvieron iguales. 
terreno y diversidad de árboles en la región también fueron incluidas en el MLO como explicativas. Sin embargo, estas variables tampoco resultaron significativas. El modelo presentado en este estudio sin las variables socio-demográficas o de percepción ajusta mejor los datos, de acuerdo al Pseudo ajustado y el Criterio de Información de Akaike (AIC, por sus siglas en inglés).

De acuerdo a la literatura, los apicultores prefieren usos de terreno que son percibidos con mayor disponibilidad de recursos florales (Bradbear, 2009; Galbraith et al., 2017). Nuestros resultados están alineados con estos hallazgos. Los resultados del MLO demuestran que los bosques son preferidos sobre otros usos de terreno, debido probablemente a los recursos florales que estos proveen. Debido a que estudios pasados han demostrado que los apicultores prefieren usos de terreno con mayores recursos florales (Galbraith et al., 2017) y nuestro estudio reveló que los apicultores prefieren los bosques y árboles con especies nativas para colocar sus colmenas, podemos concluir que los apicultores prefieren los bosques por el acceso a mayores recursos florales. Este hallazgo fue posteriormente comprobado debido a que en preguntas de seguimiento los apicultores mencionaron percibir que los bosques proveen mayores recursos florales que el pasto, café y otros usos de terreno, incluyendo las plantaciones de teca. Estos resultados indican que el cambio de uso de terreno, incluyendo el aumento en las plantaciones de teca incentivado en parte por acción local y programas a nivel nacional, no necesariamente contribuye positivamente a ciertos sectores de la agricultura, como la apicultura. De acuerdo con nuestro estudio, la percepción de la calidad de los usos de terreno influye sobre el lugar donde los apicultores deciden colocar las colmenas. Hoy día miles de hectáreas son incentivadas y utilizadas para plantaciones de teca (FONAFIFO, 2014), lo cual afecta la selección de terrenos para colocar colmenas y, consecuentemente, el ingreso de los apicultores debido a posibles cambios en los costos de viaje y producción de miel. Las abejas contribuyen al funcionamiento ecológico y a la biodiversidad floral de una región (Potts et al., 2010; Hilmi et al., 2011), por lo que crear estrategias de conservación que contribuyan al mantenimiento de las abejas es crítico.

Nuestro estudio también demostró que la calidad de la carretera juega un rol importante en la toma de decisión de los apicultores en cuanto a la selección de lugares para colocar colmenas. Este factor fue uno de los que recibió menos puntuación en los cuestionarios. Sin embargo, los resultados del MLO demuestran que, aun así, este factor es importante para los apicultores y afecta la selección de lugares. Debido a que en preguntas cerradas los apicultores mencionaron tener acceso a vehículos 4x4 para entrar al área donde se ubica el apiario, entende- 
mos que los apicultores en los cuestionarios le asignaron mayor importancia a los factores que afectan directamente las abejas y la producción de miel. Sin embargo, los apicultores prefieren tener mejores carreteras que les faciliten el trabajo y manejo de sus colmenas. Actualmente, Costa Rica está rezagado en asuntos relacionados a infraestructura, incluyendo las carreteras (World Economic Forum, 2013, 2017). Estos resultados pueden ser un incentivo para que se evalúe cómo las mejoras en carreteras pueden ayudar al desarrollo y economía del país.

En el Cuadro 3 se reportan los efectos marginales estimados que relacionan las variables explicativas con los cuatro niveles de lugares para colocar las colmenas. Por ejemplo, cada kilómetro cuadrado adicional de bosque aumenta la probabilidad de seleccionar un lugar como "más preferido" por 7.52\%. Cada kilómetro cuadrado adicional de pasto aumenta la probabilidad de seleccionar un lugar como "más preferido" por $1.76 \%$, mientras que el valor correspondiente por cada kilómetro cuadrado adicional de café es $1.79 \%$. Los lugares con carreteras que se consideren Buenas (i.e., cualquier vehículo tiene acceso al lugar durante la época seca o lluviosa) tienen más probabilidad de ser seleccionados como "más preferido" por 8.6\%. Los lugares con carreteras que se consideren Regular (i.e., solo vehículos $4 \times 4$ tienen acceso al lugar durante la época lluviosa, pero cualquier vehículo tiene acceso al lugar durante la época seca) tienen más probabilidad de ser seleccionados como "más preferido" por $3.88 \%$. Finalmente, cada diez kilómetros adicionales de distancia del hogar reducen la probabilidad de seleccionar un lugar como "más preferido" por $1.5 \%$.

\section{CONCLUSIÓN}

Este estudio encontró que los apicultores prefieren los bosques y plantaciones con árboles nativos para la apicultura debido a la variedad de recursos florales que estos proveen. Además, se encontró que

Cuadro 3.-Efectos marginales del Modelo Logit Ordenado.

\begin{tabular}{lcccc}
\hline Variables & Más preferido & $2^{\text {do }}$ preferido & $3^{\text {ro }}$ preferido & Menos preferido \\
\hline BOSQUE & $0.0752^{*}$ & $0.0197^{* 1}$ & $-0.0217^{*}$ & $-0.0732^{*}$ \\
PASTO & $0.0176^{*}$ & $0.0046^{*}$ & $-0.0051^{*}$ & $-0.0171^{*}$ \\
CAFÉ & $0.0179^{*}$ & $0.0047^{*}$ & $-0.0052^{*}$ & $-0.0174^{*}$ \\
CARRETERA_12 & $0.0388^{* *}$ & $0.0102^{* *}$ & $-0.0112^{* *}$ & $-0.0378^{* *}$ \\
CARRETERA_2 & $0.0860^{*}$ & $0.0225^{*}$ & $-0.0248^{*}$ & $-0.0837^{*}$ \\
DISTANCIA & $-0.0015^{*}$ & $-0.0004^{*}$ & $0.0005^{*}$ & $0.0015^{*}$ \\
\hline
\end{tabular}

$1 *$ Significativo al $0.01 ; * *$ Significativo al 0.1

${ }^{2}$ CARRETERA_1 = lugares con carreteras que se consideran Regular

${ }^{3}$ CARRETERA_2 = lugares con carreteras que se consideran Buenas 
aumentos en la cantidad de bosque, pasto o café, aumentan la probabilidad de seleccionar un terreno para colocar las colmenas, siendo el bosque el uso de terreno preferido por la diversidad de recursos florales. Entendemos que el gobierno de Costa Rica podría diversificar las tierras seleccionadas para plantación con árboles no-nativos, como la teca, para no afectar negativamente el sector apícola de la región, debido a que estas especies no son de interés para los apicultores.

Los resultados de este estudio demostraron que la distancia desde la casa donde reside el apicultor al lugar identificado para colocar las colmenas es uno de los factores menos importantes para los apicultores. Esto indica que los ingresos y beneficios recibidos por los recursos florales son mayores a los costos de transportación. Además, se encontró que los terrenos con mejor calidad de carretera son preferidos sobre otros lugares para colocar las colmenas. Estos resultados resaltan la necesidad de aumentar la inversión enfocada en mejorar la calidad de las carreteras debido a que el $50 \%$ de los apicultores no tiene vehículos $4 \times 4$. Es posible que el acceso a la apicultura mejore, aun cuando no haya cambios en la vegetación, si se invierte en mejorar este tipo de infraestructura. Investigaciones futuras pudieran estudiar cómo la oferta de la apicultura y de otros productos agrícolas aumentaría como resultado de mejoras en la calidad de las carreteras.

Investigaciones futuras también se pudieran enfocar en crear un plan de manejo sustentable de tal forma que el sector apícola se beneficie de los recursos florales que provee el bosque, mientras el sector agrícola se beneficie de los servicios de polinización facilitados por los apicultores. Además, el método utilizado en este estudio puede ser empleado con otros grupos de usuarios de los servicios ecosistémicos que proveen los bosques para entender mejor los impactos del PSA sobre otros sectores agrícolas. Las investigaciones sugeridas tienen el potencial de aportar al desarrollo económico local y regional, contribuyendo a un sistema social-ecológico resiliente.

\section{AGRADECIMIENTOS}

Este estudio fue financiado por NSF-IGERT proyecto \# $0903479 \mathrm{y}$ el USAID Borlaug Fellowship in Food Security beca \# A1102.2. Este proyecto fue aprobado por el Internal Review Board de la Universidad de Idaho para trabajo con sujeto humano (Proyecto 14-279). Estamos agradecidos con los apicultores de la Península de Nicoya por su participación en este estudio y con los oficiales de la oficina regional del SINAC en Hojancha por su apoyo y colaboración en esta investigación. Además, estamos agradecidos con la Dra. Troy Hall y el Dr. Chad Kooistra por su colaboración en el diseño experimental de este estudio. 


\section{LITERATURA CITADA}

Abou-Shaara, H. F., 2014. The foraging behavior of honey bees, Apis mellifera: A review. Veterinarni Medicina 59(1): 1-10.

Arrow, K., R. Solow, R. P. R. Portney, E. E. Leamer, R. Radner y H. Shuman, 1993. Report of the NOAA panel on contingent valuation. National Oceanic and Atmospheric Administration (U.S. Department of Commerce).

Bateman, I. J., R. T. Carson, B. Day, W.M. Hanemann, N. Hanley, T. Hett, M. Jones-Lee, G. Loomes, S. Mourato, E. Ozdemiroglu, D. W. Pearce, R. Sugden y S. Swanson, 2002. Economic Valuation with Stated Preference Techniques: A Manual. Edward Elgar, Massachusetts, USA.

Bradbear, N., 2009. Bees and their role in forest livelihoods: a guide to the services provided by bees and the sustainable harvesting, processing and marketing of their products. Food and Agriculture Organization of the United Nations. Rome, Italy.

Brenes-Quirós, C., 2012. Miel tica quiere endulzar más bocas. Recuperado de www.elfinancierocr.com.

Calvo-Alvarado, J., B. McLennan, A. Sánchez-Azofeifa y T. Garvin, 2009. Deforestation and forest restoration in Guanacaste, Costa Rica: Putting conservation policies in context. Forest Ecology and Management 258: 931-940.

Chagnon, M., 2008. Causes and effects of the worldwide decline in pollinators and corrective measures. Canadian Wildlife Federation. Quebec Regional Office.

Couvillon, M., F. C. Ridell-Pearce, C. Accleton, K. A. Fensome, S. K. L. Quah, E. L. Taylor y F. L.Ratnieks, 2015. Honey bee foraging distance depends on month and forage type. Apidologie 46: 61-70.

Daily, G., S. Polasky, J. Goldstein, P. M. Kareiva, H. A. Mooney, L. Pejchar, T. H. Ricketts, J. Salzman y R. Shallenberger, 2009. Ecosystem services in decision making: time to deliver. Frontiers in Ecology and the Environment 7: 21-28.

de Groot, R. S., M. A. Wilson y R. M. J. Boumans, 2002. A typology for the classification, description and valuation of ecosystem functions, goods and services. Ecological Economics 41: 393-408.

Fondo Nacional de Financiamiento Forestal (FONAFIFO), 2014. Datos históricos de las pre solicitudes recibidas: periodo 2003-2013. Recuperado de www.fonafifo.cr.gov

Galbraith, S., T. E. Hall, H. S. Tavárez, C. M. Kooistra, J. C. Ordoñez y N. A. BosquePérez, 2017. Local ecological knowledge reveals effects of policy-driven land use and cover change on beekeepers in Costa Rica. Land Use Policy, 69: 112-122.

Greenleaf, S. S., N. M. Williams, R. Winfree y C. Kremen, 2007. Bee foraging ranges and their relationship with body size. Oecologia 153(3): 589-596.

Hilmi, M., N. Bradbear y D. Mejia, 2011. Beekeeping and sustainable livelihood. Diversification booklet number 1 . Second edition. FAO. Rome, Italy.

Kasina, J. M., J. Mburu, M. Kraemer y K. Holm-Mueller, 2009. Economic benefits of crop pollination by bees: a case of Kakamega small-holder farming in western Kenya. Journal of Economic Entomology 102(2): 467-473.

Kemperman, A., A. Borgers, H. Oppewal y H. Timmermans, 2003. Predicting the duration of theme park visitors activities: an ordered logit model using conjoint choice data. Journal of Travel Research 41(4): 375-384.

Long, J. S. y J. Freese, 2001. Regression models for categorical dependent variables using Stata, Stata Press, College Station, Texas.

Madrigal-Ballestero, R., F. Alpízar y A. Schlüter, 2013. Public perception of the performance of community-based drinking water organizations in Costa Rica. Water Resources and Rural Development 1(2): 43-56.

MEA (Millennium Ecosystem Assessment), 2005. Ecosystems and human well-being: synthesis. Washington, DC: Island press.

Ostrom, E., 2009. A general framework for analyzing sustainability of social-ecological systems. Science 325: 419-422.

Pagiola, S., 2008. Payments for environmental services in Costa Rica. Ecological Economics 65: 712-724. 
Pattanayak, S. K., S. Wunder y P. Ferraro, 2010. Show me the money: do payments supply environmental services in developing countries? Review of Environmental Economics and Policy 4: 254-274.

Potts, S. G., J. C. Biesmeijer, C. Kremen, P. Neumann, O. Schweiger y W. E. Kunin, 2010. Global pollinator declines: trends, impacts and drivers. Trends in Ecology and Evolution 25(6): 345-53.

Ricketts, T. H., 2004. Tropical forest fragments enhance pollinator activity in nearby coffee crops. Conservation Biology 18(5): 1262-1271.

Serrano, M., 2005. Evaluación y planificación del manejo forestal sostenible en escala de paisaje en Hojancha, Costa Rica. Tesis de maestría. Turrialba, CR, CATIE.

Steffan-Dewenter, I., S. G. Potts y L. Packer, 2005. Pollinator diversity and crop pollination services are at risk. Trends in Ecology and Evolution 20(12): 651-652; author reply 652-653.

Stokstad, E., 2012. Field research on bees raises concern about low-dose pesticides. Science 335: 1555.

Tavárez, H., 2016. Economic valuation, land use change and ecosystem services in the Nicoya Peninsula of Costa Rica. Tesis doctoral. University of Idaho, Idaho.

Vallejo, A., I. Gutierrez, M. L. Chacon, W. V. Rooij, M. S. Davila, J. J. C. Arce y R. Villalobos, 2006. Biodiversity - human well-being linkages for teak farms in Hojancha, Nicoya Peninsula, Costa Rica. Case study commissioned by Netherlands Environmental Assessment Agency.

Vanbergen, A. J., 2013. Threats to an ecosystem service: pressures on pollinators. Frontiers in Ecology and the Environment 11(5): 251-259.

World Economic Forum, 2013. The Global Competitiveness Report 2013-2014. World Economic Forum, Geneva, Switzerland.

World Economic Forum, 2017. The Inclusive Growth and Development Report 2017. World Economic Forum, Geneva, Switzerland. 